\title{
Telenursing: A new opportunity for nurses in the digital era
}

\section{Sharma S}

Sumitra Sharma, Lecturer; Department of Nursing, Kathmandu Medical College Teaching Hospital, Kathmandu, Nepal

$I^{\text {namascas }}$ nformation technology has touched almost every aspect of our life. Health care is no exception. The use of technologies in healthcare has evolved over the years and has laid a foundation for the concept of telehealth. In broader terms, telehealth is the delivery, management and coordination of health services with the use of technology to increase access, improve outcomes and reduce costs of health care. It is found as a promising tool to address the limited capacity of heath care system for management of chronic diseases ${ }^{1}$.Most nurses are not trained or are insufficiently trained to use these tech-nologies effectively. Therefore, the potential of telehealth fails to reach full utilization. Although, telehealth is used as an umbrella term to describe the wide range of health services, the use of technology is limited within nursing profession. While telehealth in some form exists in most of the countries, telenursing is still in its infancy.

Telenursing is a novel concept. It is much beyond what patients have known in the past as counseling over the phone. It refers to the use of telecommunications technology to enhance patient care. International Council of Nurses has defined telenursing as the use of telemedicine technology to deliver nursing care and conduct nursing practice. It combines information technology to provide nursing services to the people at geographically distant locations ${ }^{2}$.This focuses on using computers, internet, telephone, telemonitoring devices and digital assessment tools to plan, assess, intervene and evaluate the outcomes of nursing care. This technique has widely expanded the scope of nursing practice in the developed nations. It is a career option for the nurses there. However, in developing countries like Nepal, it is completely a new concept that needs much awareness to put it in practice.

Address for correspondence

Sumitra Sharma,

Lecturer, Department of Nursing,

Kathmandu Medical College Teaching Hospital,

Sinamangal, Kathmandu, Nepal

E-mail: smtrsharma@gmail.com
The scope of telenursing is wide. Globally, when the health care system is widely focusing on preventive and promotive aspects of healthcare, telenursing holds many implications in health. It can open opportunities for the work of advanced practice nurses and community health nurses. Nurses can use it in providing community health services. For example, by (a) replacing faceto-face visits with e-visits via videoconferencing, (b) monitoring movements in and around the home via activity monitors, (c) monitoring vital signs such as blood pressure, heart rate via self-measurement devices, or (d) responding to personal alarms by patients to let nurses or family members know when something goes wrong.In addition, other scope of work includes palliative care, care of school children, management of patient with chronic diseases like diabetes, heart failure, depression, pulmonary diseases and even among pediatric patients. Also, this can be used in Intensive Care Unit (ICU) and Coronary Care Unit (CCU) especially on getting the instruction for the care of patients from the experts through manipulative sensors and interactive videoconferencing ${ }^{3}$.

Under staffing of nurses is a constant battle within the profession. One precise advantage to telenursing is the number of patients that can be effectively cared for per nurse. It supports in filling the gap resulting from the burden of patients and a scarcity of healthcare providers. Nurses can visit nearly twice the number of patients when travel time is not a factor ${ }^{2}$. At present, when health care costs are increasing at a rapid rate, telenursing is a compelling model to decrease unnecessary spending incurred in healthcare. Furthermore, easy access, flexible working hours, remote care services and monitoring, rapid response, opportunity for skill development are the additional positive aspects ${ }^{4}$. However, it does have limitations. Ethical dilemmas, less human contact, technological insufficiency, cost of equipment, privacy and confidentiality concerns are crucial shortcomings.

Telenursing is one of the best solutions for the multiple hindrances associated with health care delivery system in geographically diverse areas. It has found to be effective 
with the racial and culturally diverse group of population. Nepal, which is well-known for its geographical, racial and cultural diversity, telenursing can act as an effective medium to provide health services to an unreached population. Having seen multiple benefits of technology in health, the government of Nepal has implemented rural-telemedicine program in 30 district hospitals. Although nurses take part in different ways in this program, they are not focal persons in such activities ${ }^{5}$. However, study in England have shown that the adoption of new technology or the implementation of telehealth lies more on the acceptance by the frontline staff ${ }^{6}$. This suggests that unless nurses, the frontline staffs, are aware and adopt new technologies, it is hard to successfully implement telehealth in practice. Rather than being a substitute, telenursing complements existing health care network and aids in implementation of communication technology in health sector. However, acceptance of technology in nursing practice is a major challenge. Earlier study has suggested that the willingness of the nurses in telenursing will increases with their experiences in telehealth applications. Therefore, training nurses on how to integrate information and communication technology into existing healthcare pathways will certainly facilitate its acceptance.

\section{REFERENCES}

1. Van Houwelingen CTM, Moerman AH, Ettema RGA, Kort HSM, Ten Cate O. Competencies required for nursing telehealth activities: A Delphi-study. Nurse Educ Today [Internet]. 2016 [cited 2018 Jun 23];39:50-62. [ DOI]

2. Rawat G. Telenursing. Int J Curr Res [Internet]. 2017 [cited 2018 Jun 23];10(3). [ Full Text ]

3. Kawaguchi T, Azuma M, Satoh M, Yoshioka Y. Telenursing in chronic conditions. In: Health Informatics [Internet]. Springer, London; 2011 [cited 2018 Jun 26]. p. 61-74. [ DOI ]
4. Uysal H, Yeni K. Impact of health information technologies in nursing practices. ARC J Nurs Healthc [Internet]. 2016 [cited 2018 Jun 26];2(3):2455-4324. [ DOI ]

5. Bhatta R, Ellingsen G. Evaluation of the ruraltelemedicine program in Nepal: study from the selected districts [Internet]. The Artic University of Norway; 2013. [ Full Text ]

6. Taylor J, Coates E, Brewster L, Mountain G, Wessels $B$, Hawley MS. Examining the use of telehealth in community nursing: Identifying the factors affecting frontline staff acceptance and telehealth adoption. J Adv Nurs. 2015;71(2):326-37. [ PubMed ] 